\title{
Patternmaking of individualized A-line dress using computerized 3D draping method
}

\author{
KyoungOk KIM*, Koichi HIRABAYASHI ** and Masayuki TAKATERA *** \\ * Institute for Fiber Engineering (IFES), Interdisciplinary Cluster for Cutting Edge Research (ICCER), Shinshu University, Tokida \\ 3-15-1, Ueda, Nagano prefecture, 386-8567, Japan \\ kimko@shinshu-u.ac.jp \\ ** Faculty of Textile Science and Technology, Shinshu University, Tokida 3-15-1, Ueda, Nagano prefecture, 386-8567, Japan \\ 15f1046c@shinshu-u.ac.jp \\ *** Institute for Fiber Engineering (IFES), Interdisciplinary Cluster for Cutting Edge Research (ICCER), Shinshu University, Tokida \\ 3-15-1, Ueda, Nagano prefecture, 386-8567, Japan \\ takatera@shinshu-u.ac.jp
}

\begin{abstract}
This study aims to make an individualized A-line dress from three dimensional (3D) body data. Shapes of a body and an A-line dress which was fit on the body were obtained by 3D scanning. From the scanned 3D data, cross-sectional dimensions of regular intervals were obtained. Then, ratios of the maximum distance of front, back and width on transverse planes from the origin between the body and the dress were obtained as multiplication factors. A target body was deformed using the multiplication factors to construct a new dress model. Employing the proposed method, a 3D dress model was made. Using the computerized 3D draping method, the pattern was obtained and the dress was made. It was fitted on the target body while preserving the original shape dress. This method will help to make an individualized dress using 3D scan body data.
\end{abstract}

Keywords: $3 D$ draping method, individualized dress, pattern making, dress modeling

\section{INTRODUCTION}

For better wearing comfort and appearance, the demand of individualized garments is increasing. To make an individualized garment, three dimensional (3D) scanning technology is used to obtain more precise body measurements and characteristics [1]. The 3D scanning method is also used for pattern development [2]. Many researchers studied to make a clothing pattern using 3D garment surface flattening method (3D draping method) [2-4]. In those researches, garment models were generated by modeling the human body which is for tight fitting garment.

For a garment model with complex ease allowance for styling and silhouette, Zhang et al. [5, 6] proposed a method to create 3D jacket model using garment scanning data. They constructed jacket bodices for various sizes from a scanned 3D body model using the multiplication factors obtained from 3D scanned garment model and body model. Then, they successfully made the jacket bodice patterns.

This study aims to make an individualized A-line dress from 3D body scan data. A dress needs ease for a bodice and space for a skirt. Those were connected at the waist as showing curved waist line. Therefore, it is necessary to investigate the modeling method for making an individualized A-line dress.

\section{EXPERIMENTAL}

An A-line dress (bust $90 \mathrm{~cm}$, waist $74 \mathrm{~cm}$, hip $95 \mathrm{~cm}$, back length $39 \mathrm{~cm}$ ) were selected and a dress form (original body, bust $87.5 \mathrm{~cm}$, waist $63.5 \mathrm{~cm}$, hip $91.5 \mathrm{~cm}$ and back length $42 \mathrm{~cm}$ ), which the dress fits on, was selected. Those pictures are shown in Figure 1. Those 3D shapes were obtained using a 3D scanner (Body Line Scanner, Hamamatsu Photonics KK). The button and collar of the scanned dress data were deleted.

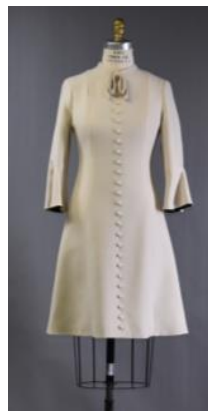

(a)

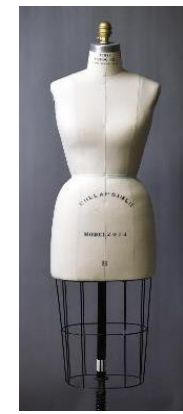

(b)

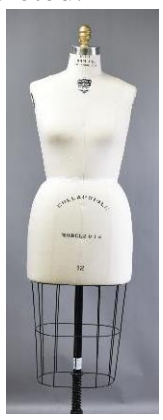

(c)
Figure 1: (a) Standard dress, (b) a standard body and (c) a target body 
From the scanned 3D data, cross-sectional dimensions of regular intervals (polyline model) were obtained as shown in Figure 2. The ratios of the maximum distance of front, back and width on transverse planes from the origin between the original body and the dress were obtained as multiplication factors [5, 6].

Another dress form (Figure 1(c), bust $94 \mathrm{~cm}$, waist $70 \mathrm{~cm}$, hips $98 \mathrm{~cm}$, and back length $44 \mathrm{~cm}$ ) was selected as a target body. The target body was deformed in vertical direction to fit positions of bust, waist, and hips lines to the positions of the original body. The target body was deformed using the multiplication factors to construct a new dress model. After applying the multiplication factors, the target body was deformed in vertical direction into the initial state. Then, the polyline model was converted into polygon model.

Using the new dress model, the dress pattern was developed using a 3D pattern making system [2]. The weft and warp grainlines were set at the similar position as the dress. Similar cutting lines were also set. Using the finished pattern and a cotton $100 \%$ fabric, a dress was made.

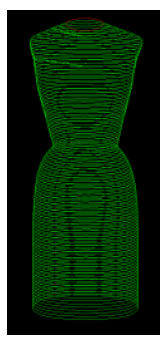

(a)

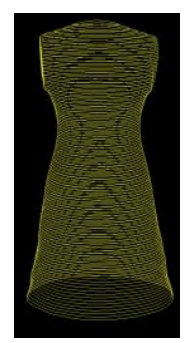

(b)
Figure 2: (a) polyline model of the body and (b) polyline model of the A-line dress

\section{RESULTS AND DISCUSSION}

Figure 3 shows the created dress models for the original body and the target body. Dress models were successfully made. Those dresses patterns were also made using a 3D pattern making system [2]. Figure 4 shows the manufactured dresses with the created patterns on the original body and the target body. Those dresses were able to keep the shape and silhouette of the A-line dress. Therefore, individualized A-line dress was successfully made using the modeling and 3D pattern making method.

\section{CONCLUSION}

By adapting the magnification factors to different bodies, it was possible to create a new dress model as keeping the shape and silhouette of the A-line dress. Using 3D patternmaking method, the patterns were also developed. The made dresses keep the shape and silhouette of the A-line dress. This method will help to make an individualized dress using 3D scan body data.

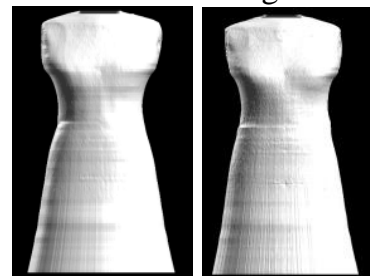

(a)

(b)

Figure 3: (a) dress model of the original body and (b) dress model of the target body

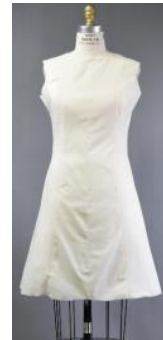

(a)

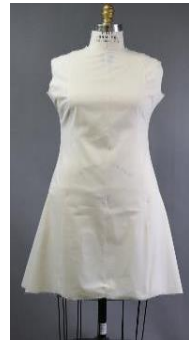

(b)
Figure 4: (a) Manufactured dress for a standard body and (b) manufactured dress for a target body

\section{REFFERENCES}

[1] KyoungOk Kim, Noriaki Innami, Masayuki Takatera, Tadaharu Narita, Midori Kanazawa and Yuji Kitazawa; Individualized male dress shirt adjustments using a novel method for measuring shoulder shape. International Journal of Clothing Science and Technology, 29(2), pp.215-225, 2017.

[2] Youngsook Cho, Takuya Komatsu, Shigeru Inui, Masayuki Takatera, Yoshio Shimizu and Hyejun Park; Individual pattern making using computerized draping method for clothing, Textile Research Journal, 76(8), pp. 646-654, 2006.

[3] In Hwan Sul and Taewook Kang, Interactive garment pattern design using virtual scissoring method, International Journal of Clothing Science and Technology, 18(1), pp. 31-42, 2006.

[4] Haruki Imaoka, Atsuo Shibuya and Noboru Aisaka; Automatic paper pattern making using mechanical development method of a curved surface on a plane surface, Sen'i Gakkaishi, 45(10), pp. 427-434, 1989.

[5] Jun Zhang, KyoungOk Kim and Masayuki Takatera; Three-dimensional garment-size change modeled considering vertical proportions. International Journal of Clothing Science and Technology, 29(1), pp. 84-95, 2017.

[6] Jun Zhang, Noriaki Innami, KyoungOk Kim and Masayuki Takatera; Upper garment 3D modeling for pattern making. International Journal of Clothing Science and Technology, 27(6), 852-869, 2015. 